\title{
Expression of Cyclin D1 and Its Relationship to Hormone Receptors and Survival in Breast Cancer
}

\author{
Senem ALANYALI, ${ }^{1}$ Levent YENIAY, ${ }^{2}$ Derya DEMIR,, ${ }^{3,4}$ Barbaros AYDIN, ${ }^{5}$ Osman ZEKIOĞLU, ${ }^{3}$ \\ Necmettin ÖZDEMIR, ${ }^{3}$ Gül KITAPÇIOĞLU, ${ }^{6}$ Ayfer HAYDAROĞLU' \\ 'Department of Radiation Oncology, Ege University Faculty of Medicine, Izmir-Turkey \\ ${ }^{2}$ Department of General Surgery, Ege University Faculty of Medicine, İzmir-Turkey \\ ${ }^{3}$ Department of Pathology, Ege University Faculty of Medicine, İzmir-Turkey \\ ${ }^{4}$ Department of Pathology, Manisa State Hospital, Manisa-Turkey \\ ${ }^{5}$ Department of Radiation Oncology, Dokuzeylül University Faculty of Medicine, İzmir-Turkey \\ ${ }^{6}$ Department of Biostatistics, Ege University Faculty of Medicine, Izmir-Turkey
}

\begin{abstract}
OBJECTIVE
The aim of the present study was to evaluate rate of cyclin D1 expression and examine its relationship to clinicopathological factors and impact on prognosis in breast cancer patients.

METHODS

Total of 85 estrogen receptor (ER)-positive breast cancer patients were included. Expression of cyclin D1, ER, progesterone receptor (PR), and Ki-67 were determined using immunohistochemistry evaluation.

RESULTS

Patients' median age was 49 years (range: $27-83$ years) and cyclin D1 was positive in $90.5 \%$ of the patients. Cyclin D1 expression was positively correlated with rate of ER positivity and Ki-67 expression $(r=0.4 ; p<0.0001$ and $r=0.3 ; p=0.001$, respectively). Five-year disease-free and overall survival (OS) rates were not different between patients with or without cyclin D1 expression ( $81 \%$ vs $79 \%$ and $93 \%$ vs $87 \% ; \mathrm{p}=0.8$ and 0.4 , respectively). High modified Bloom-Richardson grade ( $\mathrm{p}=0.04$ ), high nuclear grade ( $\mathrm{p}=0.021)$, and PR negativity $(\mathrm{p}=0.011)$ were found to be poor prognostic factors for OS rate in univariate analysis.
\end{abstract}

\section{CONCLUSION}

In this study, cyclin D1 was not found to be prognostic factor; however, it is correlated with ER positivity and Ki-67 expression in breast cancer patients.

Keywords: Breast cancer; cyclin D1; prognostic factors.

Copyright $\odot$ 2017, Turkish Society for Radiation Oncology

\section{Introduction}

Breast cancer is the most common cancer among women. It is estimated that in 2016 the number of newly diagnosed breast cancer in the United States will be 246.660 and the number of estimated deaths due to breast cancer will be 40.450 . [1] With the widepread use of screening programs and advances in treatment (targeted drugs, modern radiotherapy (RT) techniques) 
cure rates of breast cancer is improved.[2] The treatment of breast cancer is patient-oriented so the sequencing of surgery, radiotherapy, chemotherapy, and targeted therapies might show differences in every patient.[3]

There are well known and widely used prognostic and predictive factors of breast cancer include patient age, stage, presence of lymph node metastasis and tumor biology. [4] There are elaborate studies on molecular markers which might have prognostic importance in breast cancer. Identification of prognostic and predictive molecular markers will lead to more aggressive treatment for high-risk patients and prevent unnecessary treatment and side effects in low-risk patients. It is thought that the survival difference observed between cases with similar clinical and pathological features is due to many factors not yet clarified in tumor biology. Recently, researchers have reported that breast cancer is a heterogeneous disease and composed of different subtypes (luminal A, luminal B, Her-2, and basal). In our daily practice identification of subtypes informs clinicians about the treatment response and local regional/distant recurrence patterns of the patient.[5]

However, intensive investigations have been carried out on signal transduction mechanisms involved in cell cycle.[6,7] Among these, cyclins are one of the most studied markers. Cyclins, cyclin-dependent kinases (CDKs) and inhibitors (CDKI) are proteins that directly control the cell cycle. Overexpression of Cyclin D1 and Cyclin E lead to impaired control of the cell cycle. It has been suggested that overexpression of Cyclin D1 and Cyclin $\mathrm{E}$ in breast cancer might be prognostic factors. [8-10] There are reports that cyclins may play a role in some of the hormone sensitive breast cancer cases that are resistant to tamoxifen and the mechanism of action of tamoxifen is affected by the deterioration of control of cell cycle.[11,12] However there is no consensus in the literature on the role of Cyclin D1 in breast cancer biology and its prognostic and predictive value.

We aimed to evaluate Cyclin D1 expression and to investigate its prognostic significance in our breast cancer patient population.

\section{Materials and Methods}

Between 1994 and 2004, breast cancer patients treated with surgery, and irradiated in our department were evaluated. Among them, 85 of the patients who had ER positive breast cancer and the paraffin blocks are available in our Pathology Department were included to the analysis.
External RT was applied to the breast / chest wall and peripheral lymphatics with a total of 50-60 Gy at 5-6 weeks with 2 Gy fractions per day; chemotherapy (CT) were administered before or after RT or by sandwich method. Hormonal therapy (antiestrogen / aromatase inhibitor) was administered to patients for 5 years after RT. Patients were followed up once every 3 months for the first 2 years, every 6 months for $2-5$ years after treatment and annually thereafter.

\section{Immunohistochemical analysis}

Macroscopic data of the operation materials were obtained from archival records of Pathology Department. The operation material for each patient was stained with hemotoxylin-eosin for 6-8 hours after $10 \%$ neutral formalin fixation. In our study, sections of 4-5 micrometer thickness were taken and immunohistochemical examination was performed on sections made from paraffin embedded tissue samples with formalin fixation. Estrogen (clone SP1, Neomarkers, 1: 250 dilution), progesterone (clone SP2, Neomarkers, 1: 250 dilution) p 53 (Clone DO-7, Dako, 1:50 dilution), cyclin-D1 (clone SP4, Neomarkers, 1: 100 dilution) primer antibodies were investigated. As the immunohistochemical staining system, we used a biotin-free, HRP multimer-based, hydrogen peroxide substrate and 3,3'-diaminobenzidine tetrahydrochloride (DAB) chromogen (UltraView ${ }^{\text {Tx }}$ Universal DAB Detection Kit, Catalog number 760-500, Ventana Medical Systems, Tucson, AZ). Tissue sections were taken from electrostatically charged slides (X-traTM, Surgipath Medical Industries, Richmond, Ill., USA) and dried at $60^{\circ} \mathrm{C}$ for at least two hours. All immunohistochemical staining procedures, including deparafinization and antigen removal, were performed in the BenchMark XT fully automatic immunohistochemical staining device. Only the primary antibodies estrogen (SP1), progesterone (SP2), cyclin D1 (SP4) were manually instilled and incubated at $37^{\circ} \mathrm{C}$ for 32 minutes. In the device, the process was terminated by dehydration of hematoxylin and bluerization solution, cross-sectioning of xylenes, transparency of xylene and closure of coverglass.

Histological evaluation was performed using a standard light microscope (Olympus Bx50; x40 magnification, $0.54 \mathrm{~mm}$ diameter, Ocular magnification: x10) without using a special ocular grating. As positive controls, serial sections for breast carcinomas detected by immunohistochemistry for $100 \%(+3)$ for estrogen and progesterone and mantle cell lymphoma for cyclin D1 were used. 


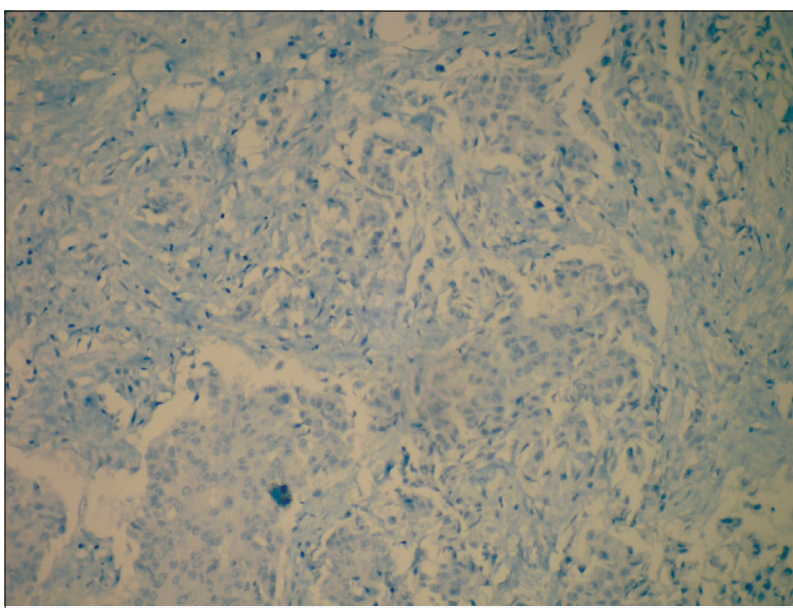

Fig. 1. Negative staining of Cyclin D1 immunohistochemically (Cyclin D1, x200).

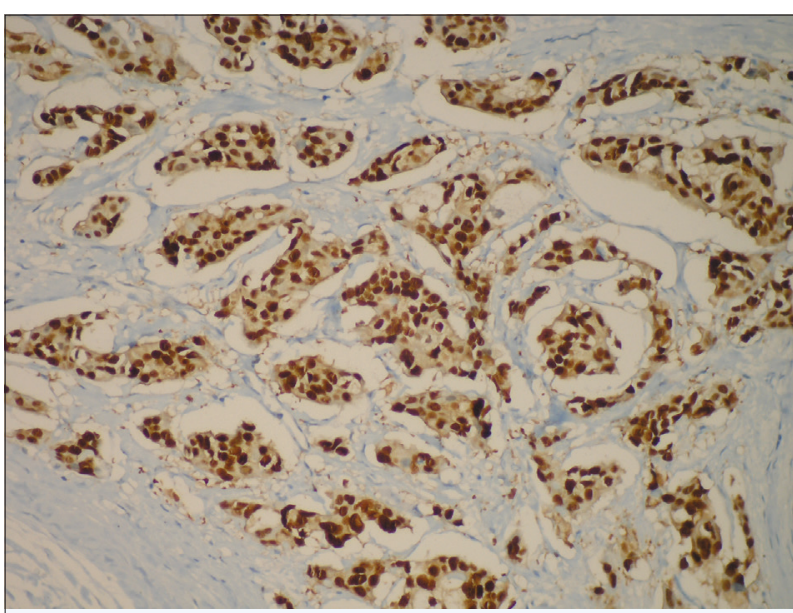

Fig. 2. Positive staining of Cyclin D1 immunohistochemically (Cyclin D1, x200).

In immunohistochemical examination, nuclear staining of estrogen, progesterone and cyclin-D1 was accepted as positive. The ratio of tumor cells showing nuclear positivity and the intensity of staining were evaluated as 1, 2 and 3 positive for ER and PR. The ratio of tumor cells showing nuclear positivity for Cyclin D1 and the intensity of staining were evaluated as 1,2 and 3 positive. Nuclear positivity for Ki-67 and p53 was considered significant. Positive tumor cell ratios were given by choosing the areas that were most intense. The threshold value for positive staining was accepted as $10 \%$. Negative and positive expressions of Cyclin D1 are shown in Figures 1 and 2 respectively.

For statistical analysis, data were entered in SPSS 13.0 (Statistical Package for the Social Sciences) soft-

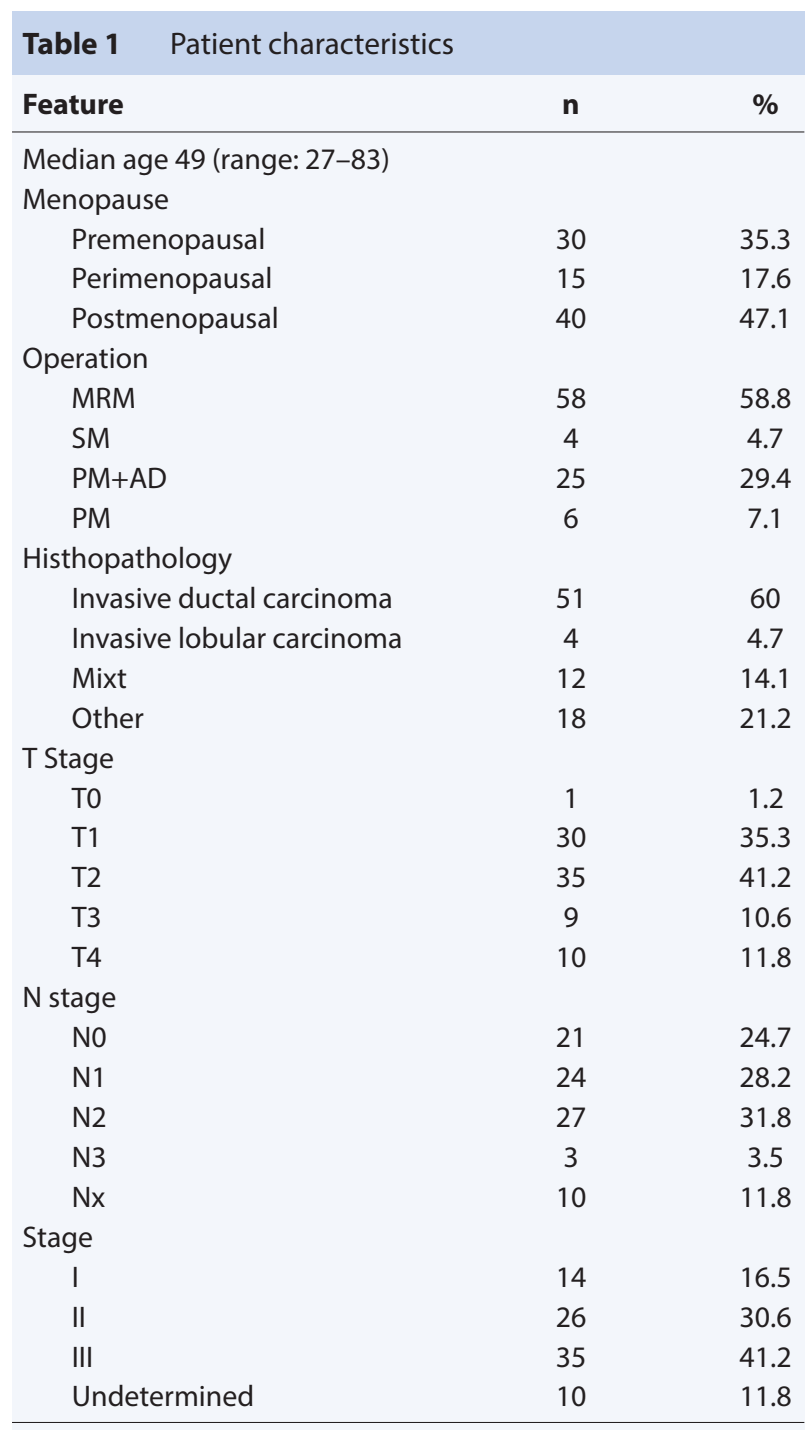

MRM: Modified radical mastectomy; SM: Simple mastectomy; PM+AD: Partial mastectomy+axillary dissection; PM: Partial mastectomy.

ware, general characteristics of cases were defined with basic statistical methods and Kaplan Meier, log-rank and chi-square methods were used for correlation and survival analysis.

\section{Results}

Median age of the patients was 49 (range: 27-83) and approximately $2 / 3$ of the cases were stage II and III. The most common type of surgery is modified radical mastectomy and general characteristics of the patients are summarized in Table 1. In 10 patients axillary intervention was not performed and were accepted as Nx. Axillary dissection was performed in $68(80 \%)$ 


\begin{tabular}{|c|c|c|}
\hline Marker & $\mathbf{n}$ & $\%$ \\
\hline \multicolumn{3}{|l|}{ p53 } \\
\hline Negative & 68 & 80 \\
\hline Positive & 17 & 20 \\
\hline \multicolumn{3}{|l|}{ Ki-67 } \\
\hline Negative & 36 & 42.4 \\
\hline Positive & 49 & 57.6 \\
\hline \multicolumn{3}{|c|}{ Estrogen receptor } \\
\hline$(+)$ & 18 & 21 \\
\hline$(++)$ & 10 & 35 \\
\hline$(+++)$ & 37 & 43 \\
\hline \multicolumn{3}{|c|}{ Progesterone receptor } \\
\hline Negative & 30 & 35.2 \\
\hline$<\% 10$ & 8 & 9.4 \\
\hline$\geq \% 10$ & 47 & 55.2 \\
\hline \multicolumn{3}{|c|}{ Progesterone receptor } \\
\hline$(-)$ & 30 & 35.2 \\
\hline$(+)$ & 28 & 32.9 \\
\hline$(++)$ & 19 & 22.4 \\
\hline$(+++)$ & 8 & 9.4 \\
\hline \multicolumn{3}{|l|}{ Cyclin D1 } \\
\hline Negative & 5 & 5.9 \\
\hline$<\% 10$ & 3 & 3.5 \\
\hline$\geq \% 10$ & 77 & 90.5 \\
\hline \multicolumn{3}{|l|}{ Cyclin D1 } \\
\hline$(-)$ & 5 & 5.9 \\
\hline$(+)$ & 4 & 4.7 \\
\hline$(++)$ & 12 & 14.1 \\
\hline$(+++)$ & 64 & 75.3 \\
\hline
\end{tabular}

cases and the median number of dissected lymph node was 17 (range: 10-44). In 7 cases number of dissected lymph nodes was below 10 and accepted as axillary sampling. The most common histopathological type is invasive ductal carcinoma. Lymphovascular invasion was present in $16(18.8 \%)$, perineural invasion in $4(4.7 \%)$, pectoral muscle invasion in $2(2.4 \%)$ and fascia invasion in 4 cases (4.7\%). Histological grades were 1,2 and 3 in 7 (8.2\%), 31 (36.5\%) and $47(55.3 \%)$ and nuclear grades were 1, 2 and 3 in 12 (14.1\%), 55 (64.7\%) and 14 (16.5\%) patients respectively. According to the Modified Bloom Richardson grading, grade 1,2 , and 3 cases were 26 (30.6\%), 41 (48.2\%) and 15 (17.6\%) respectively.

All cases were ER positive and immunohistochemical results are presented in Table 2. Cyclin D1 expression was positively correlated with ER-positive rate and Ki-67 expression $(r=0.4, p<0.0001$ and $r=0.3, p=0.001$, respectively). In the follow-up period of median 60 months (range: 6-156 months), 13 (15.3\%) cases had distant
Table 3 Evaluation of prognostic factors for disease free and overall survival

\begin{tabular}{|c|c|c|c|c|}
\hline Variable & 5 y DFS (\%) & $\mathbf{p}$ & 5 y OS (\%) & $\mathbf{p}$ \\
\hline \multicolumn{5}{|l|}{ Cyclin D1 } \\
\hline$\leq \% 10$ & 79 & $\mathrm{p}=0.8$ & 87 & $p=0.4$ \\
\hline$>\% 10$ & 81 & & 93 & \\
\hline \multicolumn{5}{|l|}{ Cyclin D1 } \\
\hline$(-)$ & 80 & $\mathrm{p}=0.8$ & 50 & $\mathrm{p}=0.2$ \\
\hline$(+)$ & 75 & & 100 & \\
\hline$(++)$ & 82 & & 100 & \\
\hline$(+++)$ & 82 & & 92 & \\
\hline \multicolumn{5}{|l|}{ ER } \\
\hline$(+)$ & 75 & $p=0.4$ & 90 & $p=0.9$ \\
\hline$(++)$ & 81 & & 96 & \\
\hline$(+++)$ & 85 & & 89 & \\
\hline \multicolumn{5}{|l|}{ PR } \\
\hline$(-)$ & 68 & $\mathrm{p}=0.2$ & 82 & $p=0.011$ \\
\hline$(+)$ & 83 & & 100 & \\
\hline$(++)$ & 88 & & 100 & \\
\hline$(+++)$ & 100 & & 85 & \\
\hline \multicolumn{5}{|c|}{ Modified Bloom } \\
\hline \multicolumn{5}{|c|}{ Richardson grade } \\
\hline 1 & 93 & $\mathrm{p}=0.1$ & 100 & $p=0.04$ \\
\hline 2 & 72 & & 93 & \\
\hline 3 & 79 & & 78 & \\
\hline \multicolumn{5}{|l|}{$\mathrm{HG}$} \\
\hline 1 & 100 & $p=0.6$ & 100 & $p=0.4$ \\
\hline 2 & 78 & & 94 & \\
\hline 3 & 75 & & 88 & \\
\hline \multicolumn{5}{|l|}{ NG } \\
\hline 1 & 77 & $\mathrm{p}=0.1$ & 100 & $p=0.021$ \\
\hline 2 & 85 & & 94 & \\
\hline 3 & 56 & & 73 & \\
\hline
\end{tabular}

HG: Histological grade; NG: Nuclear grade; ER: Estrogen receptor; PR: Progesterone receptor; DFS: Disease free survival; OS: Overall survival.

metastases and $3(3.6 \%)$ cases had local recurrences. There was no difference in the 5-year disease-free (DFS) and overall survival (OS) rates $(81 \%$ vs $79 \%$ vs $84 \%$ vs $87 \%, \mathrm{p}=0.8$ and $\mathrm{p}=0.4$ ) among patients with Cyclin D1 positive and negative staining. In our study, negative prognostic factors for OS were found to be high Modified Bloom Richardson grade $(\mathrm{p}=0.04)$, high nuclear grade $(\mathrm{p}=0.021)$ and progesterone receptor negativity $(\mathrm{p}=0.011)$, while any prognostic factor could not be detected for disease free survival in our study (Table 3).

\section{Discussion}

Carcinogenesis process is caused by numerous mutations affecting biological events such as cell viabil- 
ity, growth control, and differentiation. During cancer development, tumor cells gain many phenotypic properties. These changes cause rapid and unlimited proliferation of tumor cells and their invasion to the surrounding tissues. Serial mutations of protooncogenes and tumor suppressor genes contribute to the formation of malignant phenotypes through different mechanisms. Oncogenic mutations targeting signaling pathways and signaling proteins are also common. The changes in the signal transmission affect the control of the growth and/or survival functions of the cell. Thus, oncogenic signal transduction plays an active role in tumor development, invasion and metastasis processes.[13]

Cyclins, cyclin-dependent kinases (CDKs) and inhibitors (CDKI) are proteins that directly control cell cycle. Cyclin D1 is located on the chromosome 11q13 and encodes the $36 \mathrm{kD}$ Cyclin D1 protein. Cyclin D1 plays a role in regulating the G1 phase in normal cell cycle. With the induction of cyclin D1, the duration of the G1 phase is shortened and the number of cells passing through the G1 phase increases. Therefore the overexpression of Cyclin D1 is resulted with uncontrolled growth in tumor cell. In normal cells, Cyclin D1 behaviour varies according to external stimuli such as growth factors, and estrogen.[14]

Cyclin D1 levels were found to increase by $20 \%$ in benign and premalign (atypical ductal hyperplasia) breast lesions, by $70-80 \%$ in ductal carcinoma in situ lesions and by $30-70 \%$ in invasive breast cancer.[15] Cyclin D1 expression rate was found higher in ER (+) breast cancer. In our study, it was determined that Cyclin D1 expressed in $90 \%$ of the cases. This expression ratio is higher than the studies in the literature, because only ER (+) cases are included in our analysis.

Cyclin D1 is one of the 21 genes evaluated in the Oncotype DX gene test using for the patient-specific treatment selection for breast cancer. While some studies reported increased expression as a good prognostic factor in estrogen receptor positive patients, other studies found that it was associated with early recurrence and poor prognosis. Pelosio et al. investigated the association of Cyclin D1 expression with ER and PR receptor status and its prognostic significance in 180 breast cancer patients with axillary lymph node metastasis. They found that ER and PR positivity was higher in tumors with Cyclin D1 expression and the increased Cyclin D1 nuclear staining is related with high recurrence free survival rates.[16]

Seshadri et al. investigated the relationship between CCND1 gene amplification and its relation with the clinicopathologic features and prognosis of 1014 breast cancer cases. The researchers found that CCND1 gene amplification was observed in hormone receptor positive tumors and this amplification was significantly correlated with ER and PR positivity. With a median follow-up of 66 months, they found that CCND1 amplification was not associated with breast cancer recurrence and breast cancer-related mortality in the whole group. However this amplification was associated with increased recurrence rates in node-negative and ER (+) groups.[17]

$\mathrm{Xu}$ et al. performed a meta-analysis including approximately 9000 cases in 33 trials and found that for patients with overexpression of Cyclin D1, HR was 1.13 $(\mathrm{p}=0.35), 1.25(\mathrm{p}=0.12)$ and $1.04(\mathrm{p}=0.76)$ for overall survival, progression free survival and distant metastases free survival respectively. However, specifically for ER (+) breast cancers, overexpression of Cyclin D1, HR was $1.67(\mathrm{p}=0.00)$ for overall survival, and researchers found that Cyclin D1 expression is related with poor prognosis of ER (+) patients.[18]

Among the published literature, there is no consensus on the prognostic value of Cyclin D1. In our ER (+) breast cancer patient population we did not demonstrate Cyclin D1 expression as a prognostic factor for DFS and OS. We think that the inconsistency regarding the prognostic significance of Cyclin D1 expression might be related with the heterogeneity among the published studies, possible genetic polymorphisms in CCND1 encoding Cyclin D1, and possible subtypes with different functions of Cyclin D1 that are yet to be identified.

Warwick et al. analysed the prognostic factors in their 20 year follow-up study, and concluded that the importance of tumor grade at the time of diagnosis have a lasting influence on survival.[19] Debled et al. analyzed prognostic factors of early distant recurrence in hormone receptor-positive, postmenopausal breast cancer patients who receive adjuvant tamoxifen therapy. Among 715 breast cancer patients, a distant recurrence occured in 38 patients (5.3\%) within the first 3 years of tamoxifen therapy. In this analysis, modified Scarff-Bloom-Richardson grade 3 was the only significant predictive factor of early recurrence (hazard ratio, 3.72; $\mathrm{p}<.001)$. [20] In our analysis we also reiterate that high nuclear grade and high modified Bloom Richardson grade are unfavorable prognostic factors for OS.

Since we don't have the HER-2 information of the patient, we cannot perform prognostic analysis according to molecular subtypes. However as already shown in other studies, we also found that ER (+) PR (+) tu- 
mors tend to have better survival rates than ER (+) and $\mathrm{PR}(-)$ tumors.[21,22]

Jirström et al. suggest that Cyclin D1 is associated with ER and PR receptors and may interact with antiestrogen therapy. They immunohistochemically assessed CCND1 gene amplification in 500 breast cancer patients. It has been reported that there is an agonistic effect between tamoxifen treatment and CCND1 amplification in ER-positive tumors.[23] Gillett et al. also showed that breast cancer cases receiving tamoxifen have better response and better survival rates in the presence of Cyclin D1 expression.[10] In our study, we also found that Cyclin D1 expression was positively correlated with ER positivity and also with Ki-67 expression.

Ahnström et al. investigated the prognostic significance of overexpression of Cyclin D1 and C-erbB2 in 230 breast cancer patients randomized to tamoxifen, chemotherapy and radiotherapy arms and found that the prognostic value of C-erbB2 expression was greater in patients with overexpressed Cyclin D1. In ER positive cases, it was found that cases with no expression or poor expression of Cyclin D1 did not benefit from tamoxifen treatment. Investigators have indicated that Cyclin D1 may be a marker for tamoxifen resistance.[24]

Understanding the potential role of Cyclins, cyclin dependent kinases (CDKs) and inhibitors (CDKI) in breast cancer has made them an important target. In the recently published PALOMA-1/TRIO-18 trial, palbociclib, an inhibitor of Cyclin-dependent kinases 4 and 6 , was used. In the cell cycle, G1 to $S$ phase transition is controlled by cyclin dependent kinases (CDK4 and 6) and palbociclib is an oral CDK 4/6 inhibitor. In this Phase II trial, ER-positive metastatic breast cancer patients were randomised to letrazol versus letrazol and palbociclib arms. At 2.5 years follow-up, median progression-free survival was 20.2 months in the palbociclib arm, whereas only 10.2 months in the letrazol arm, and this difference was statistically significant. [25] This study reiterates the importance of defining and targeting the molecular mechanisms of breast cancer.

Our study is valuable in determining the expression and prognostic role of Cyclin D1 in the ER positive breast cancer population in Turkey and but limited with the number of cases. In our daily clinical practice, we believe that the identification of cases with Cyclin D1 expression and administration of targeted therapies will increase response and cure rates of the patients.

\section{Acknowledgments}

We would like to thank Professor Serra Kamer for her valuable comments for editing the manuscript.

\section{Disclosure Statement}

The authors declare no conflicts of interest.

\section{References}

1. http://www.cancer.org/acs/groups/content/@research/ documents/document/acspc-047079.pdf. Accessed November 21, 2016.).

2. Whelan TJ, Julian J, Wright J, Jadad AR, Levine ML. Does locoregional radiation therapy improve survival in breast cancer? A meta-analysis. J Clin Oncol 2000;18(6):1220-9.

3. Recht A, Come SE, Henderson IC, Gelman RS, Silver B, Hayes DF, et al. The sequencing of chemotherapy and radiation therapy after conservative surgery for earlystage breast cancer. N Engl J Med 1996;334(21):135661.

4. Goldhirsch A, Wood WC, Gelber RD, Coates AS, Thürlimann B, Senn HJ. Progress and promise: highlights of the international expert consensus on the primary therapy of early breast cancer. Ann Oncol 2007;18(7):1133-44.

5. Sørlie T, Perou CM, Tibshirani R, Aas T, Geisler $\mathrm{S}$, Johnsen $\mathrm{H}$, et al. Gene expression patterns of breast carcinomas distinguish tumor subclasses with clinical implications. Proc Natl Acad Sci USA 2001;98(19):10869-74.

6. Tonini G, Schiavon G, Fratto ME, Vincenzi B, Santini D. Hormono-biological therapy in metastatic breast cancer: preclinical evidence, clinical studies and future directions. Expert Opin Biol Ther 2008;8(2):221-34.

7. Arnold A, Papanikolaou A. Cyclin D1 in breast cancer pathogenesis. J Clin Oncol 2005;23(18):4215-24.

8. McIntosh GG, Anderson JJ, Milton I, Steward M, Parr $\mathrm{AH}$, Thomas MD, et al. Determination of the prognostic value of cyclin D1 overexpression in breast cancer. Oncogene 1995;11(5):885-91.

9. Michalides R, Hageman P, van Tinteren H, Houben L, Wientjens E, Klompmaker R, et al. A clinicopathological study on overexpression of cyclin D1 and of p53 in a series of 248 patients with operable breast cancer. $\mathrm{Br}$ J Cancer 1996;73(6):728-34.

10. Gillett C, Smith P, Gregory W, Richards M, Millis R, Peters G, et al. Cyclin D1 and prognosis in human breast cancer. Int J Cancer 1996;69(2):92-9.

11. Van Diest PJ, Michalides RJ, Jannink L, van der Valk P, Peterse HL, de Jong JS, et al. Cyclin D1 expression in invasive breast cancer. Correlations and prognostic 
value. Am J Pathol 1997;150(2):705,11.

12. Umekita Y, Ohi Y, Sagara Y, Yoshida H. Overexpression of cyclinD1 predicts for poor prognosis in estrogen receptor-negative breast cancer patients. Int J Cancer 2002;98(3):415-8.

13. De Vita VT, Hellman S, Rosenberg SA, eds. Cancer: Principles \& Practice of Oncology, Philadelphia, PA: Lippincott Williams Wilkins, Seventh Edition 2005;73-164.

14. Bates S, Peters G. Cyclin D1 as a cellular proto-oncogene. Semin Cancer Biol 1995;6(2):73-82.

15. Weinstat-Saslow D, Merino MJ, Manrow RE, Lawrence JA, Bluth RF, Wittenbel KD, et al. Overexpression of cyclin D mRNA distinguishes invasive and in situ breast carcinomas from non-malignant lesions. Nat Med 1995;1(12):1257-60.

16. Pelosio P, Barbareschi M, Bonoldi E, Marchetti A, Verderio P, Caffo O et al. Clinical significance of cyclin D1 expression in patients with node-positive breast carcinoma treated with adjuvant therapy. Ann Oncol 1996;7(7):695-703.

17. Seshadri R, Lee CS, Hui R, McCaul K, Horsfall D J, Sutherland RL. Cyclin DI amplification is not associated with reduced overall survival in primary breast cancer but may predict early relapse in patients with features of good prognosis. Clin Cancer Res 1996;2(7):1177-84.

18. Xu XL, Chen SZ, Chen W, Zheng WH, Xia XH, Yang HJ, et al. The impact of cyclin D1 overexpression on the prognosis of ER-positive breast cancers: a metaanalysis. Breast Cancer Res Treat 2013;139(2):329-39.

19. Warwick J, Tabàr L, Vitak B, Duffy SW. Time-dependent effects on survival in breast carcinoma: results of 20 years of follow-up from the Swedish Two-County
Study. Cancer 2004;100(7):1331-6.

20. Debled M, MacGrogan G, Brouste V, Mathoulin-Pelissier S, Durand M, Mauriac L. Prognostic factors of early distant recurrence in hormone receptor-positive, postmenopausal breast cancer patients receiving adjuvant tamoxifen therapy: results of a retrospective analysis. Cancer 2007;109(11):2197-204.

21. Nishimukai A, Yagi T, Yanai A, Miyagawa Y, Enomoto Y, Murase K, et al. High Ki-67 Expression and Low Progesterone Receptor Expression Could Independently Lead to a Worse Prognosis for Postmenopausal Patients With Estrogen Receptor-Positive and HER2-Negative Breast Cancer. Clin Breast Cancer 2015;15(3):204-11.

22. Sun JY, Wu SG, Li FY, Lin HX, He ZY. Progesterone receptor loss identifies hormone receptor-positive and HER2-negative breast cancer subgroups at higher risk of relapse: a retrospective cohort study. Onco Targets Ther 2016;9:1707-13.

23. Jirström K, Stendahl M, Rydén L, Kronblad A, Bendahl PO, Stål O, et al. Adverse effect of adjuvant tamoxifen in premenopausal breast cancer with cyclin D1 gene amplification. Cancer Res 2005;65 (17):8009-16.

24. Ahnström M, Nordenskjöld B, Rutqvist LE, Skoog L, Stål O. Role of cyclin D1 in ErbB2-positive breast cancer and tamoxifen resistance. Breast Cancer Res Treat 2005;91(2):145-51.

25. Finn RS, Crown JP, Lang I, Boer K, Bondarenko IM, Kulyk SO, et al. The cyclin-dependent kinase 4/6 inhibitor palbociclib in combination with letrozole versus letrozole alone as first-line treatment of oestrogen receptor-positive, HER2-negative, advanced breast cancer (PALOMA-1/TRIO-18): a randomised phase 2 study. Lancet Oncol 2015;16(1):25-35. 